\section{Automated, Robotic Preparation of Vitrified Samples for 2D and 3D Cryo Electron Microscopy}

\author{
Peter. M. Frederik ${ }^{1}$ and Marc M.H. Storms ${ }^{2}$
}

1. University Maastricht, EM-unit, Universiteitssingel 50, 6229 ER Maastricht, The Netherlands, peter.frederik@elmi.unimaas.nl

2. FEI Company, Achtseweg Noord 5, 5600 KA Eindhoven, The Netherlands,marc.storms@nl.feico.com

\section{Introduction}

Fundamental research within the scope of cell and structural biology as well as nano-technology is increasingly focusing on unraveling interactive biological and biochemical processes and pathways at the macromolecular level. For this, high resolution transmission electron microscopy (TEM) is indispensable. Of paramount importance is the three-dimensional visualization of macromolecular structures and molecular machines in their native hydrated state. Their physical fixation within ultra-thin vitrified ice layers is the crucial starting point. Although vitrification seems easy to achieve it can be quite demanding to realize, considering the various sample properties and vitrification parameters that are significant for acquiring proper results: temperature, relative humidity, blotting frequency and pressure, as well as wait times in between the various steps.

TEM forms an image from electrons transmitted through the sample. Thus, the primary requirement for sample preparation is that the sample be thin enough to permit transmission - generally a few hundred nanometers or less for biological materials. In general, thinner is better, since it increases the fraction of electrons that are transmitted and improves the signal to noise ratio of the image. For vitrified films, where the specimen is not physically sectioned, the thickness of the film may ultimately be limited by the size of the object to be studied. For example, when studying liposome size and shape, small liposomes will yield better results since they will permit thinner films.

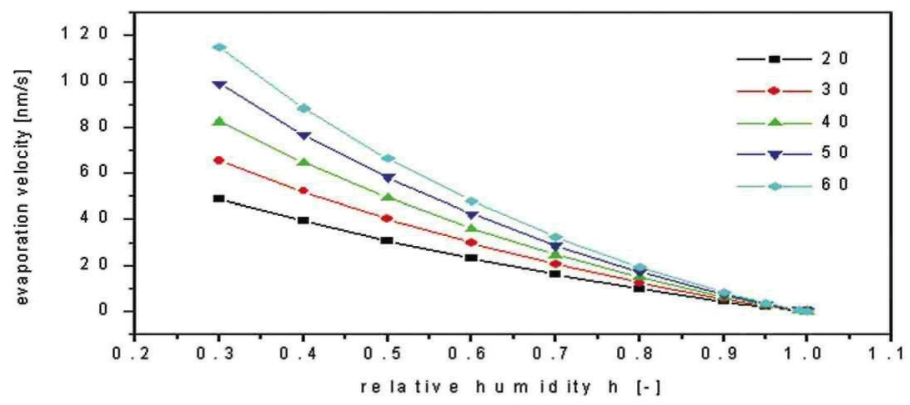

Figure 1: Evaporation velocity (loss of film thickness in $\mathrm{nm} / \mathrm{sec}$ ) versus relative humidity at temperatures ranging from $20^{\circ}$ to $60^{\circ} \mathrm{C}$.

In the vitrification process a sample is transformed from a three dimensional aqueous solution, usually at near-biological conditions of temperature and concentration, to a pseudo-two dimensional thin film at cryo temperatures. The vitrification process itself is extremely fast, typically taking only a few microseconds (Dubochet et al. 1992), but the procedures that must occur prior to that, as the sample is extracted from solution and applied to the TEM sample grid, occur over a time span of many seconds and provide ample opportunity for interaction between the sample and the environment. Hence the perfect control of important conditions is critical to the success of the preparation process (Frederik and Hubert 2005).

\section{Heat and Mass Transfer Prior to Vitrification}

Sample preparation for cryo-electron microscopy involves a well defined sequence of steps: application of the sample to a specimen grid, blotting away excess fluid, draining/thinning the film, and finally, vitrification, achieved by plunging the thin sample into a liquid coolant (typically ethane, propane or nitrogen). Once the solution is applied to the grid it has an extremely high surface to volume ratio and its behavior is dominated by processes that occur at the air/liquid interface. The most important of these interactions are heat and mass transfer that result from evaporation. These may be analyzed as a dew point effect and the specimen will ultimately achieve an equilibrium temperature dependent on the temperature and relative humidity of the sample preparation environment.

Although the specimen solution and the preparation chamber may initially be at the same temperature (typical laboratory ambient of $20^{\circ} \mathrm{C}$ and $40 \%$ relative humidity $(\mathrm{rH})$ ) evaporation from the sample reduces its temperature to the dew point where, in the absence of additional heat input, evaporation and condensation are in equilibrium. At the dew point, the partial vapor pressure of water in the environment equals the saturated vapor pressure of the dew point temperature. It can be shown that for conditions typical for sample preparation, the sample will attain the dew point temperature very quickly, ranging from a few tenths of a second for relatively thick films, to less than a tenth of a second for film thicknesses below $500 \mathrm{~nm}$. The cooling of the sample to the dew point creates a temperature differential across which heat continues to flow so that there is a steady movement of mass (evaporating water) from the sample into the environment. The evaporation rate depends on temperature and relative humidity and is independent of film thickness.

Figure 1 plots evaporation velocity as a function of relative humidity for five different temperatures (20 ${ }^{\circ}-60^{\circ} \mathrm{C}$ ). Clearly, it will be easier to achieve a consistent film thickness at higher relative humidity where the evaporation velocity is lower. Furthermore it is clear that thin film preparation under room conditions $\left(\right.$ e.g. $20^{\circ} \mathrm{C}$ and a relative humidity of $40 \%$ ) may result in an appreciable loss of water - in two seconds a film will lose 80 $\mathrm{nm}$ of its thickness. Evaporation also affects the solute and particle concentrations of the film. An $80 \mathrm{~nm}$ reduction in the thickness of a 160 nm film essential doubles the concentration. Changes in

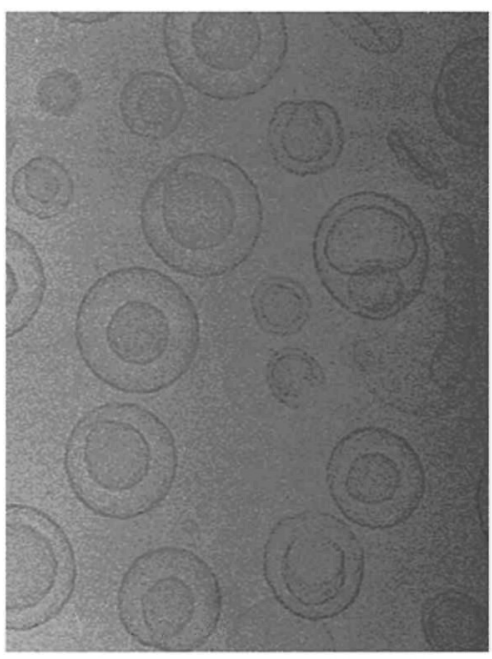

Figure 2 Osmotic effects. The spherical vesicles collapse into vase-like structures as the internal volume shrinks in response to an osmotic gradient established by the evaporation of solvent during thin film preparation at $25^{\circ}$ and $40 \% \mathrm{rH}$. (DOTAP/cholesterol in glucose/300 mosm) 


\section{X-ray pulses need not be cold, wet or slow!}

So, in 1996 we did something about that - we changed the world of EDX

\section{XFlash}

From RÖNTEC

The originator of SDD $X$-ray detector technology

for SEM, TEM,

and Microprobe

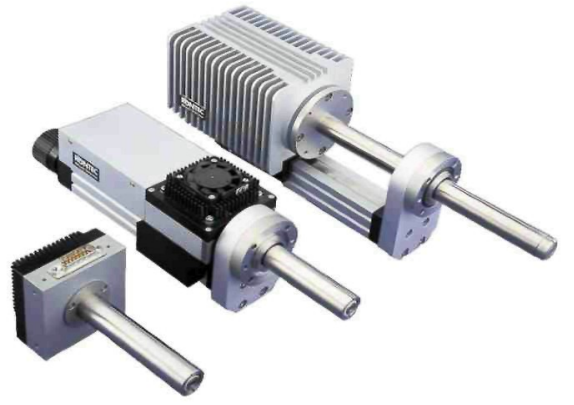

Now, the ultra-fast RÖNTEC "SD3"

will make you a believer

\section{Acquisition rates up to 275,000 cps!}

\section{The Premium High Performance LN2 Free SDD X-ray Detector is here} Detection of boron to uranium - $127 \mathrm{eV}$ resolution at $\mathrm{Mn}$ and $58 \mathrm{eV}$ at $\mathrm{C}$

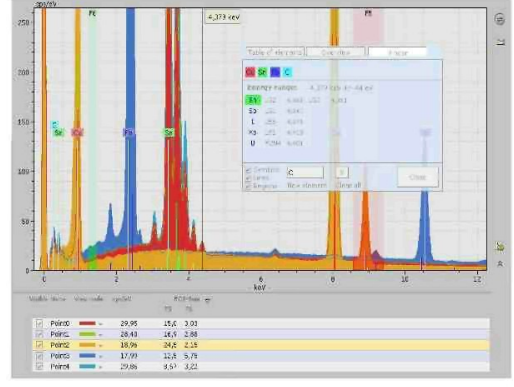

Multiple Spectra Acquisition

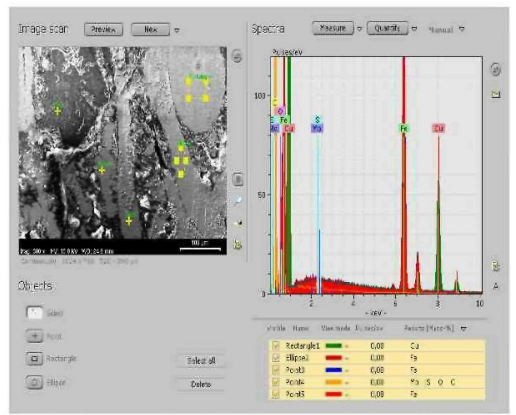

Automatic Multipoint Analysis

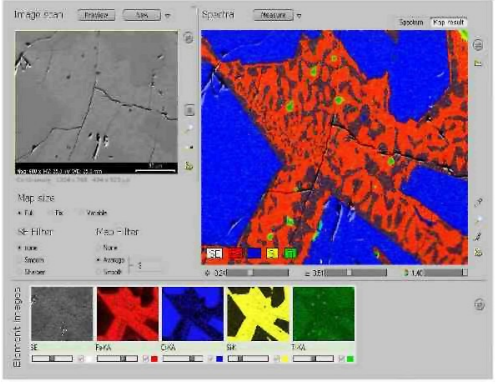

Ultrafast X-ray Mapping

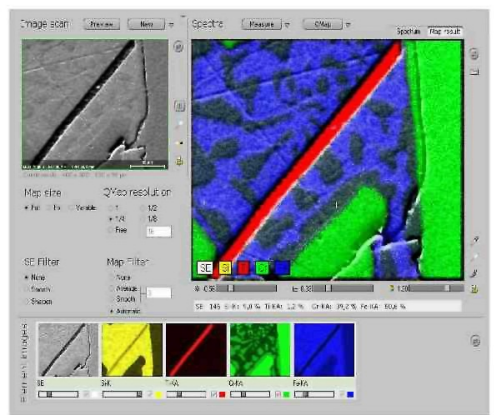

Detail rich Quant Mapping

Proven with over 1,400 RÖNTEC SDD's sold

$\checkmark$ Excellent sensitivity and spectral stability

$\checkmark$ Rugged \& Reliable - no icing or defrosting as with Si(Li)'s

$\checkmark$ No vibration or microphonics

$\checkmark$ Convenient and easy to use

$\checkmark$ Instant on/off - no waiting for cool down

$\checkmark$ No Consumables - Maintenance Free

$\checkmark$ New $L$ and $M$ line data for improved quant

$\checkmark$ Built with the quality and pride you expect

$\checkmark$ QuanTax is integrated with HKL EBSD

From the leader in SDD detector technology

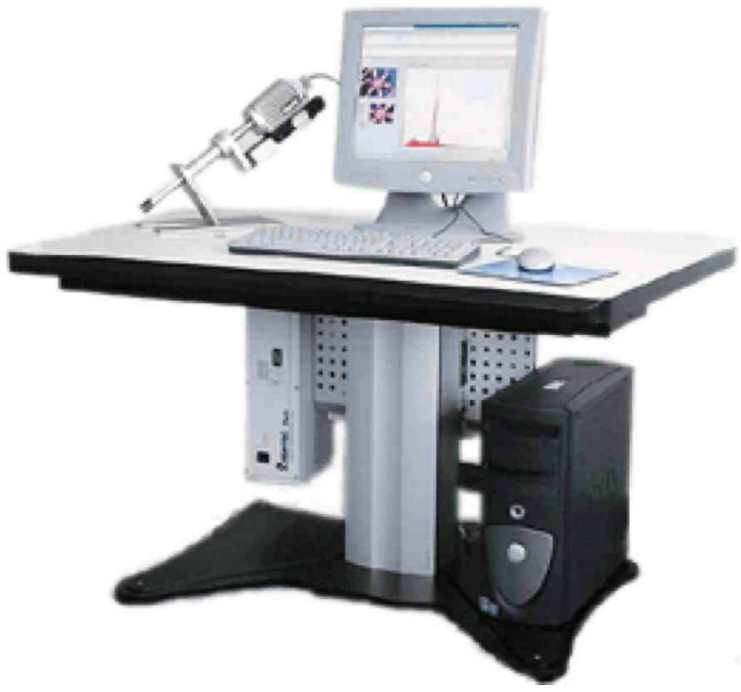

RÖNTEC "QuanTax" EDX System

\section{N. \\ RÖNTEC}

RÖNTEC USA Inc.

Carlisle, MA 01741

Tel: (978) 266-2900

sales@rontecusa.com

www.rontec.com

A subsidiary of RÖNTEC AG 


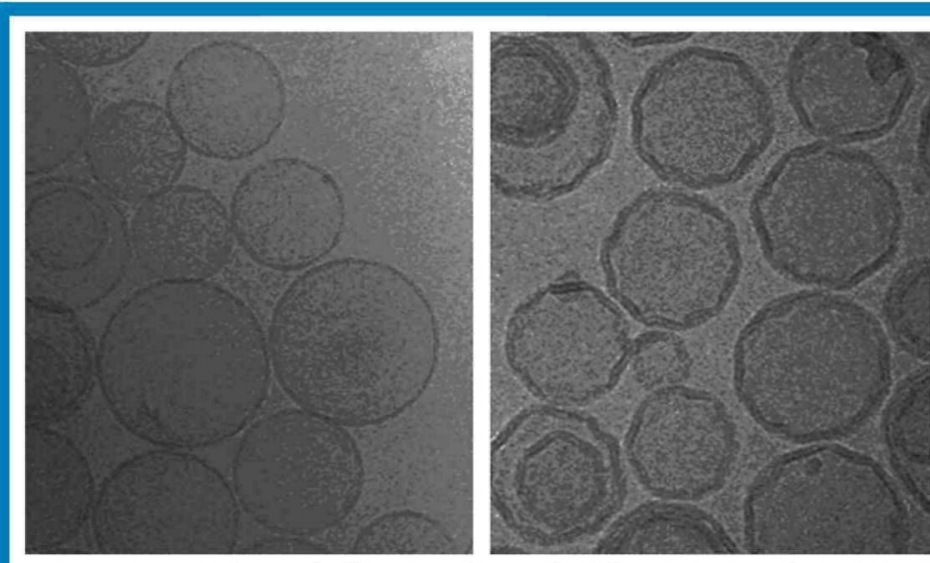

Figure 3 Thermal effects. Vesicles on the left were prepared at $50^{\circ} \mathrm{C}$ and $100 \% \mathrm{rH}$, on the right, $12 \% \mathrm{rH}$. Low relative humidity reduces the sample temperature and results in the formation of $P_{\beta^{\prime}}$ (ripple) phase. High $r H$ preserves the $L_{\alpha}$ structure. (DPPC in distilled water). Images courtesy of Frederik and Hubert, 2005

solute concentration (osmotic effects - fig 2) may induce changes in the very structures one wishes to observe. Finally, higher relative humidity reduces the temperature differential between the environment and the evaporatively cooled sample, making it easier to avoid temperature induced changes (thermal effects - fig 3) in the structure. It should be apparent that higher relative humidity in the preparation chamber combined with precise timing of the various steps of the pre-vitrification preparation will yield enhanced control of the temperature, thickness and concentration of the sample up to the instant of vitrification, and avoid thermally and osmotically induced changes in structure.

\section{Automated, robotic vitrification}

The vitrification robot or Vitrobot $\mathrm{TM}^{\mathrm{T}}$ is a guillotine-like device. The vertical motion is provided by a driven rod to which standard forceps clamping a sample grid are attached. The blotting action is provided by blotting paper attached to rotating disks that are arranged to close on the sample like "clapping hands". The disks rotate to provide a fresh blotting surface for each clap and the duration and pressure of each action are precisely controlled. The entire apparatus is enclosed in an environmental chamber. A shutter at the bottom of the chamber provides access to the rod to load new forceps/grids and to plunge the sample into the coolant.

The operational sequence begins with lowering the rod under stepper motor control and attaching the forceps and grid through the opened shutter. The rod retracts and the shutter closes. The sample solution is loaded onto the grid through a hole in the chamber using a pipette to extract solution from a vial inside the chamber, and deposit a controlled amount (typically $3 \mu \mathrm{l}$ ) on the grid. The grid is positioned for blotting and a controlled sequence of blotting actions is performed. The grid is allowed to drain/thin for a controlled period of time. Finally the rod is disengaged from the stepper motor and pneumatically propelled (approx. $2 \mathrm{~m} / \mathrm{sec}$ ) through the shutter into the coolant. After vitrification the coolant reservoir engages the rod/forceps/sample assembly and can be removed from the system to permit further manipulation and transfer to the TEM using conventional cryotransfer tools. All aspects of the preparation sequence are controlled by a computer system that includes an easy-to-use operator interface.

The environmental chamber can be heated or cooled through a range of $4^{\circ}$ to $60^{\circ} \mathrm{C}$ by using a Peltier cooling/heating element. An ultrasonic mist generator provides forced humidification and can achieve $100 \% \mathrm{rH}$ in about one minute. A serendipitous effect of working at high humidity is the elimination of minor temperature differences within the chamber. Water condenses on cooler surfaces, raising their temperatures, and evaporates from warmer surfaces, lowering their temperatures.

The same technology (and the reasoning behind) can be applied to the cryo-EM observation of thin films prepared from suspensions in organic solvents (Butter et al., 2003) and this further broadens the scope of cryo-EM.

\section{Three Dimensional Reconstruction}

Molecular biologists (and others who would analyze the structure of organic macromolecules) are confronted with a number of difficult challenges. The structures that they seek to analyze offer inherently low contrast, being composed primarily of lighter elements, often embedded in a close packed matrix of similarly composed molecules - as in the interior of a cell. The samples are delicate and easily damaged by the electron beam. The structures are often flexible, such that the same molecule may assume a range of configurations. In fact, the ability to change configuration in response to specific stimuli is an important functional characteristic of many biological molecules. Finally, the molecules often function as part of larger complexes of several molecules.

Conventional methods for determining molecular structure
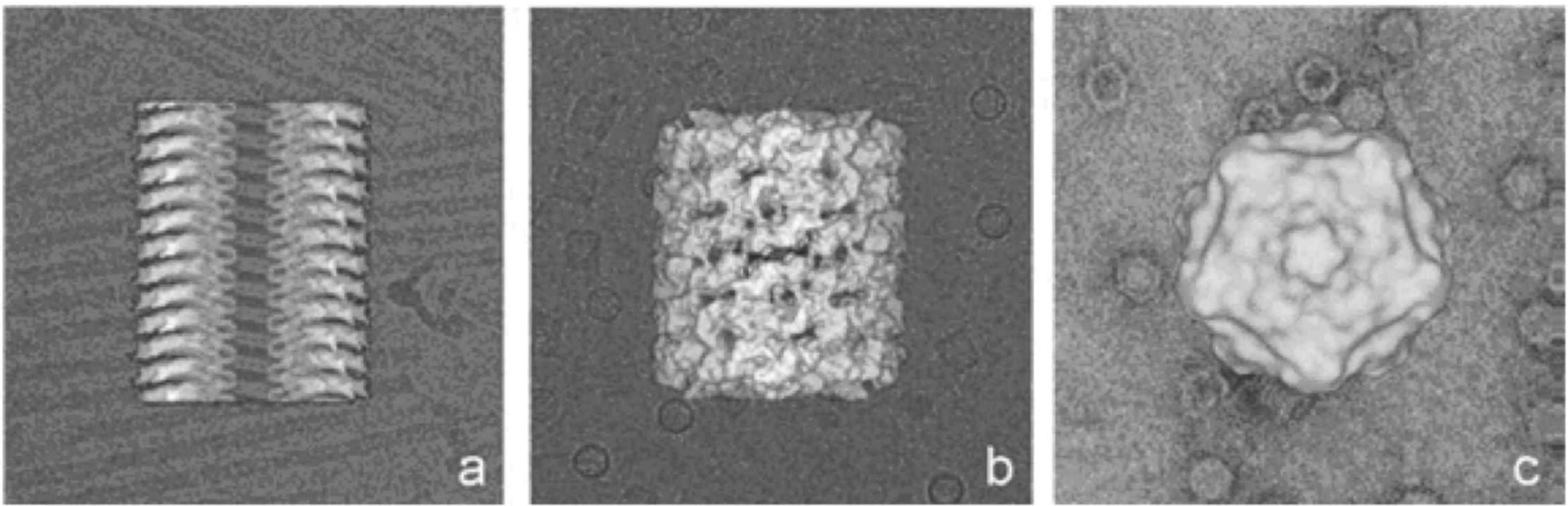

Figure 4 a) Tobacco mosaic virus (TMV), b) keyhole limpet hemocyanin, c) cowpea mosaic virus (CPMV) structures determined by SPA. Images courtesy of Carragher et al., Journal of Synchrotron Radiation 11:83-85, (c) $2004 \mathrm{IUCr}$ 


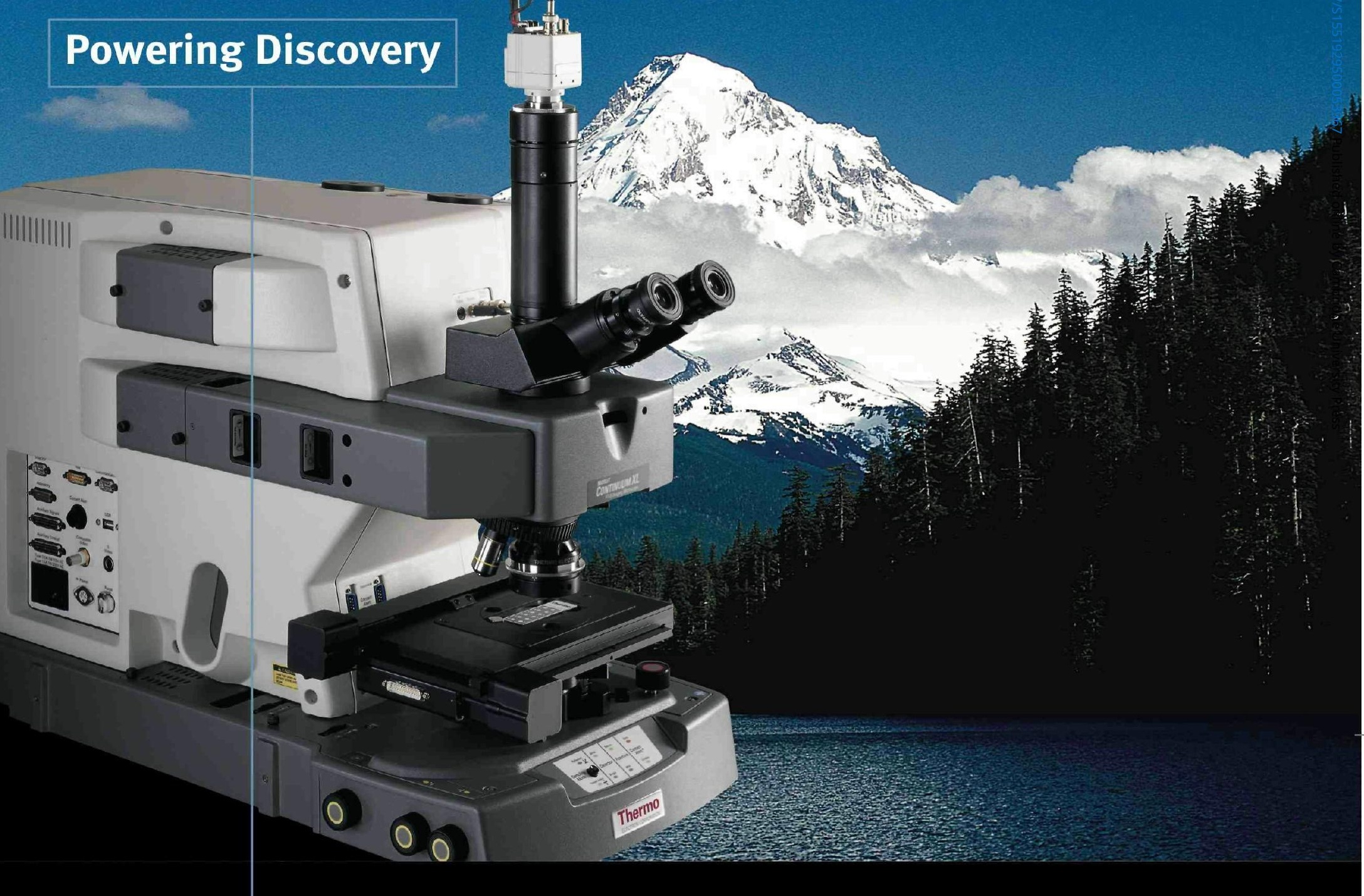

Nicolet Continuum XL FT-IR Imaging Microscope
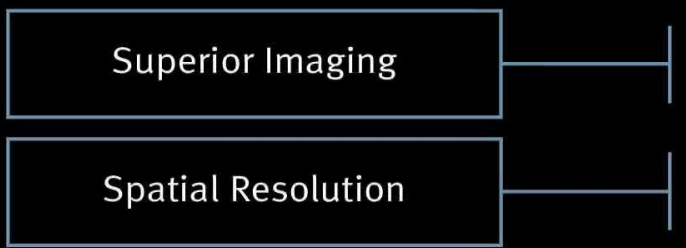

\section{Greater Clarity}

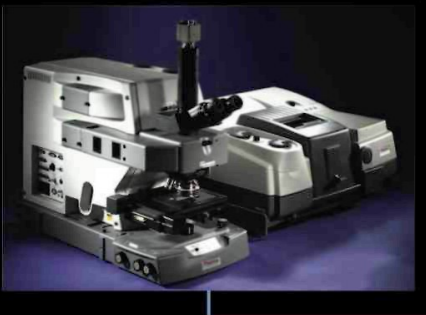

Thermo Electron introduces the Nicolet ${ }^{\text {TM }}{\text { Continu } u m^{T M}}^{\text {TL }} \mathrm{L}$, a research-quality FT-IR microscope with the most advanced imaging available.

Unlock even your most mystifying sample. The innovative imaging design provides full spectral range with unprecedented image quality. Improve your throughput. A dual-masking remote aperture provides a continuous view of the sample while simultaneously collecting the purest spectral data.

Soar beyond current limitations. Couple the revolutionary imaging of this instrument with the power of Nicolet FT-IR spectrometers.

See the future of imaging today. Contact Thermo Electron at 1-800-532-4752, email analyze@thermo.com or visit www.thermo.com/ftir 


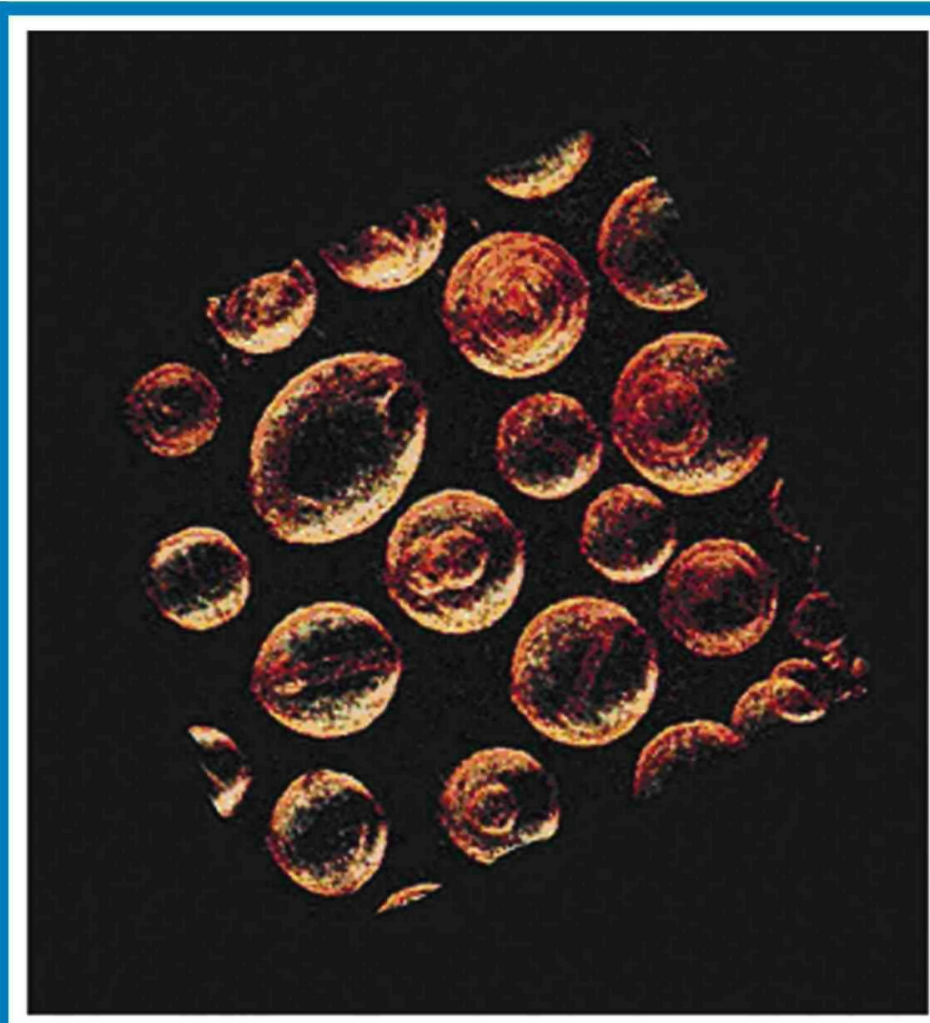

Figure 5 Doxorubicin-loaded liposomes reconstructed using electron tomography. Image courtesy of Frederik and Hubert 2005 (also see Lasic et al 1995).

include X-ray diffraction of crystals (XRD) and nuclear magnetic resonance (NMR) spectroscopy. Though these techniques are both capable of atomic level resolution each has limitations that restrict its application to a relatively narrow class of molecules. XRD requires the sample to be crystallized. It can take months or years to find conditions under which a particular molecule will crystallize, and once a crystal has been formed the question remains whether the crystallized structure accurately reflects the in vivo structure. NMR does not require crystallization but does require a large number of identical molecules. The analysis of NMR data is computationally intense and structural determination can take months for even a relatively simple molecule.

CryoEM cannot match the atomic scale structural resolution of these techniques but is broadly applicable to most biological materials. In many ways CryoEM may be considered complementary to XRD and NMR. It does not require crystallization and can be used to observe molecules in their native biological environment. It works particularly well with larger molecules and molecular complexes, as in the determination of tertiary and quaternary protein structure. Its ability to literally freeze molecular motion allows users to study structural dynamics and interactions between molecules.

All TEM images are a two dimensional projections of three dimensional structures. There are two fundamentally different approaches to recreate the $3 \mathrm{D}$ structure from the projections: socalled single particle analysis (SPA) and electron tomography. As mentioned previously, TEM images of biological material inherently have a low contrast because of the composition of the material. This situation is compounded by the restrictions placed on total exposure to the electron beam in order to avoid beam induced damage. The information contained in low-dose images is often

impossible to discern visually. Both SPA and tomography average the information from a large number of images to enhance the contrast and signal-to-noise ratio of the final reconstruction.

Single particle analysis is a somewhat misleading terminology since in fact it relies on the combination of images from a large number of identical particles in arbitrary orientations. The images are first selected and categorized into groups with similar orientations. The images in each group are then averaged to refine the resolution and contrast of that projection. Finally the averaged projections from all groups are back projected to recreate the $3 \mathrm{D}$ structure of the sample. SPA works best with rigid structures since any flexibility interferes with both the grouping and averaging procedures. In SPA each particle is only exposed to the beam once, relaxing, to some extent, the restrictions on beam current. The total number of images included in the analysis is limited only by the number of particles available for imaging. A typical analysis may include tens of thousands of images. Theoretically the resolution can be increased arbitrarily by increasing the number of images, though the large number of images required imposes a practical limit on resolution of one to two nanometers. The entire process from image acquisition through analysis has been automated, providing results in as little as twenty-four hours. Figure 4 shows the structures of three different biological samples as determined by SPA.

\section{Electron} tomography acquires multiple images of a single particle as it is rotated incrementally around an axis. Ideally the particle should be rotated through a full $360^{\circ}$. However, practical considerations limit the rotation to $\pm 70^{\circ}$, leaving a region of uncertainty in the resulting reconstruction. Because the same particle is exposed repeatedly to the beam, limiting the electron dose is a primary concern. The resulting images have extremely low

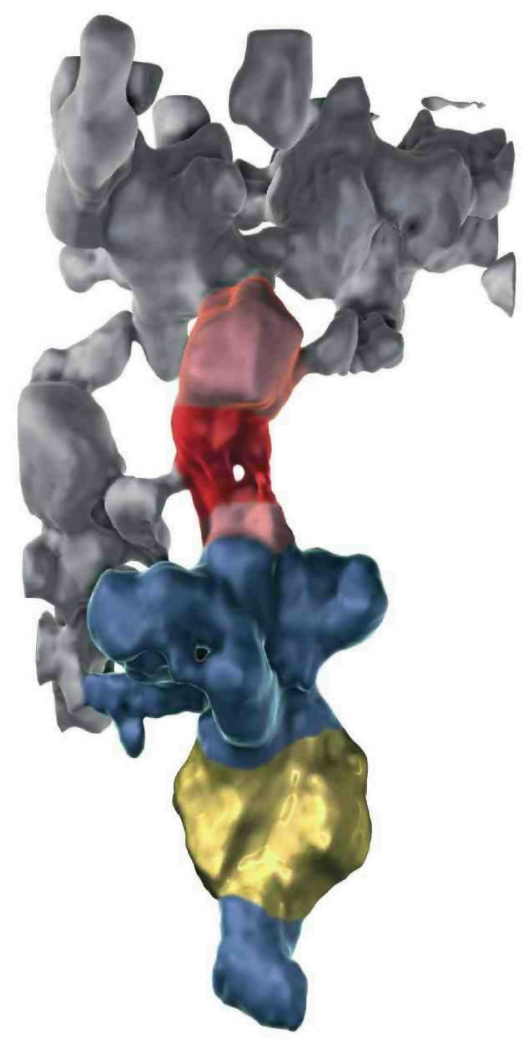

Figure 6 Membrane receptor. Neurotrophin receptor p75 (light red) reconstructed using electron tomography. The images used in this reconstruction were acquired in situ, although the membrane itself is not included in this rendering. Grey structures are other membrane proteins. Yellow and blue are gold labeled antibodies used to locate the receptor. Image courtesy of Sidec Technologies, as published in Elands and Hax, Current Drug Discovery, 2004. 


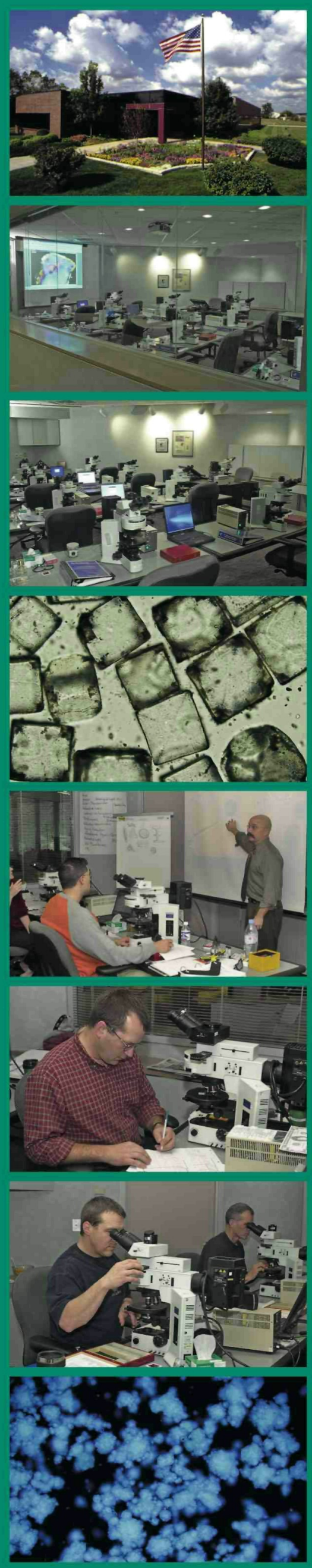

if COLLEGE OF MICROSCOPY

Located in Westmont, Illinois, the College of Microscopy is an institution for specialized instruction and education, whose goal is to advance the knowledge and understanding of light and electron microscopy for materials analysis.

One of the advantages of attending a course at the College of Microscopy is that our courses are taught primarily by experienced senior staff scientists from

McCrone Associates, Inc. who solve micro-analytical problems on a daily basis. That's why it's true when we say,

\section{"Learn From Experience...}

\section{Learn From The Experts."}

\section{Polarized Light Microscopy}

COM100: Polarized Light and Chemical Microscopy

Feb. 13-17, 2006 April 3-7, 2006

COM160: Techniques of Optical Crystallography July 17-21, 2006

\section{Electron Microscopy}

COM200: Scanning Electron Microscopy

March 27-31, 2006 Oct. 16-20, 2006

COM240: Advanced Electron Microprobe Training

Oct. 2-6, 2006 Taught by Peter McSwiggen, Ph.D.

\section{Imaging/Software}

COM500: Image Analysis Workshop

Taught by John Russ, Ph.D.

April 25-27, 2006

\section{Sample Preparation}

COM300: Microscopic Particle Handling

April 24-28, 2006

COM310: Pharmaceutical and Medical Devices

Jan. 18-19, 2006

COM311: Polymers, Paint, and Coatings

Feb. 22-23, 2006

COM312: Forensics and Trace Evidence

COM313: Microelectronic Devices and Materials

March 8-9, 2006

April 11-12, 2006

\section{Special Applications}

COM400: Microscopical Examination of Forensic Trace Evidence Particles (Part 1)

Aug. 21-25, 2006

COM410: Microscopical Identification of Pharmaceutical

July 24-28, 2006 Materials and Contaminants

COM430: Microscopical Identification of White-

Jan. 23-27, 2006 Powder Unknowns (Part 1)

May 8-12, 2006

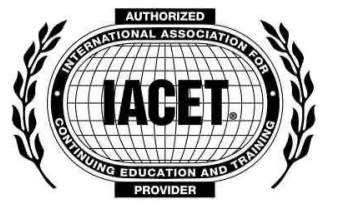

IACET CEUs awarded for all courses completed with the College of Microscopy

College of Microscopy $\bullet 850$ Pasquinelli Drive, Westmont, Illinois 60559 www.collegeofmicroscopy.com 
contrast and low signal-to-noise ratios. Special computational techniques are used to enhance the information content of the images, which are then back-projected to reconstruct the sample structure. Tomography typically provides structural resolutions of a few nanometers, but has the advantage of looking at a single particle frozen in time. Particle flexibility does not interfere with the analysis. By analyzing a collection of such particles tomography can provide information about structural variability and eventually structural dynamics. The same capability permits investigations of particle interactions and concomitant structural changes.

\section{Time resolved CryoEM}

The speed and precise timing of robotic vitrification offers the prospect of time resolved CryoEM. A number of mechanisms have been proposed to initiate interactions among molecular components that can then be captured at some subsequent instant in time to study the progress of the interaction. The mechanisms include spraying the interacting components on the grid, using electrostatic fields to deflect a spray onto the grid, and blotting dry components onto the sample with impregnated blotting paper. Another innovative approach uses "caged "compounds; bound substances (e.g. ATP, Ca, protons) that are released upon the interaction of a flash of UV light. Using this technique it may be possible to attain a minimum time interval between flash and vitrification as short as $1 \mathrm{msec}$.

\section{Conclusion}

Automated, robotic vitrification provides tight control of temperature and humidity in the sample preparation environment, as well as precise control of timing for all steps in the process. Temperature and humidity in the sample preparation environment determine the rate of heat and mass transfer from the sample. Operating at high $\mathrm{rH}$ reduces evaporation velocity and, when combined with precise timing, improves both the speed and consistency of the vitrified thin film specimens, and avoids osmotic and thermal effects that may induce structural changes in the sample. Vitrification provides samples that are in a nearly pristine natural state, and that lend themselves well to the analysis of three dimensional structure using single particle analysis and electron tomography. The ability of vitrification to freeze samples in a moment of time holds further promise in the development of time-resolved CryoEM which would allow researchers to follow the progress of molecular processes over time courses as brief as a few milliseconds. The combination of robotic vitrification and CryoEM will likely play an important role in our growing understanding of macromolecular processes.

\section{References}

1 Dubochet, J., Adrian, M., Chang, J.J., Homo, J.C., Lepault, J., McDowall, A.W. and Schultz, P. (1992). Cryo-electron microscopy of vitrified specimens. Q. Rev. Biophys. 21, 129-228

2 Frederik, P.M. and Hubert, D.H.W. (2005). Cryo-electron microscopy of liposomes. Methods in Enzymology vol 391, 431-448.

3 Butter, K, Bomans, P.H.H., Frederik, P.M., Vroege, G.J. and A.P. Philipse (2003). Direct observation of dipolar chains in iron ferrofluids by cryogenic electron microscopy. Nature Materials 2, 88-91.

4 Carragher et al., (2004) Journal of Synchrotron Radiation 11:83-85.

5 Lasic, D.D., Ceh, B., Stuart M.C., Guo, L., Frederik P.M. and Barenholz,Y. (1995). Transmembrane gradient driven phase transitions within vesicles: Lessons for Drug Delivery. BioChim. Biophys. Acta 1239, 145-156.

6. Elands, J. and W.H.A. Hax (2004). Current Drug Discovery, 15-20.

\section{Microscopy Microanalysis}

Table of Contents Preview

Volume 11, Number 6, December 2005

\section{BIOLOGICAL APPLICATIONS}

Ultrastructure, Histochemistry, and Mineralization Patterns in the

Ecdysial Suture of the Blue Crab, Callinectes sapidus

Carolina Priester, Richard M. Dillaman, and D. Mark Gay

Microwave Processing for Sample Preparation to Evaluate Mitochon-

drial Ultrastructural Damage in Hemorrhagic Shock

Gary D. Josephsen, Kelly A. Josephsen, Greg J. Beilman, Jodie H. Taylor, and Kristine E. Muiler

Effects of Inhibitors of $\Delta^{24(25)}$-Sterol Methyl Transferase on the

Ultrastructure of Epimastigotes of Trypanosoma cruzi

Marina V. Braga, Filippo Magaraci, Silvia Orenes Lorente, lan Gilbert, and Wanderley de Souza

Characterization of Hydroxyapatite by Electron Microscopy V. Rodríguez-Lugo, J. Sanchez Hernández, Ma. J. Arellano-Jimenez, P.H. Hernández-Tejeda, and S. Recillas-Gispert

\section{MATERIALS APPLICATIONS}

Interaction of Oxide Surfaces with Water: Environmental Transmission

Electron Microscopy of $\mathrm{MgO}$ Hydroxylation Marija Gajdardziska-Josifovska and Renu Sharma

Atomic Structure of $\beta$-Tantalum Nanocrystallites

Karsten Tillmann, Andreas Thust, Andreas Gerber, Martin P. Weides, and Knut Urban

\section{MICROANALYSIS}

Misidentification of Major Constituents by Automatic Qualitative Energy Dispersive X-ray Microanalysis: A Problem that Threatens the Credibility of the Analytical Community Dale E. Newbury

PAPERS FROM THE EUROPEAN MICROBEAM ANALYSIS SOCIETY WORKSHOP IN BLED, SLOVENIA

The Change of the LMM Auger Spectra in 3d-Metals Due to Oxidation and Its Correlation with the Change of the Atomic Magnetic Moment Olga R. Zheltysheva, Dmitry V. Surnin, Dmitry E. Guy, Faat Z. Gil'mutdinov, Yuri V. Ruts, and Vladimir I. Grebennikov

AES Investigation of Inhomogenous Metal-Insulator Samples Gábor Dobos, György Vida, Zoltán Tóth, and Katalin Josepovits

Electron Microscopy Studies of Potassium Sodium Niobate Ceramics Darja Jenko, Andreja Bencan, Barbara Malic, Janez Holc, and Marija Kosec

Inelastic Mean Free Path Data for Si Corrected for Surface Excitation Gábor Tamás Orosz, György Gergely, Sándor Gurbán, and Miklós Menyhard

Indexed in Chemical Abstracts, Current Contents, BIOSIS, and MEDLINE (PubMed)

MSA members receive both Microscopy Today and Microscopy and Microanalysis FREE! 
